УДК 519.876.5

\title{
АВТОМАТИЗАЦИЯ СРЕДСТВ ИЗМЕРЕНИЙ \\ В УЧЕБНОМ ПРОЦЕССЕ ТЕХНИЧЕСКОГО ВУЗА
}

Мельников Михаил Алексеевич

студент

Гордяскина Татьяна Вячеславовна

к.ф.-м.н.

Лебедева Светлана Владимировна

К.T.H.

Мерзляков Владимир Иванович

К.T.H.

доценты кафедры Радиоэлектроники ФГБОУ ВО «ВГУВТ»

Аннотация: В работе рассматривается пример автоматизации средств радиоизмерений в рамках учебного процесса. Приводится пример разработки цифрового характериографа, используемого в натурных экспериментах при снятии вольт-амперных характеристик нелинейных радиоэлементов (полупроводниковых приборов). Предлагается методика статистической обработки результатов измерений в Microsoft Office Excel.

Ключевые слова: Цифровизация средств измерений, характериограф, вольт-амперная характеристика, полупроводниковые диоды, цифровое моделирование.

\section{AUTOMATION OF MEASURING INSTRUMENTS \\ IN THE EDUCATIONAL PROCESS OF A TECHNICAL UNIVERSITY}

Melnikov Mikhail Alekseevich Gordyaskina Tatiana Vyacheslavovna Lebedeva Svetlana Vladimirovna Merzlyakov Vladimir Ivanovich 
Abstract: The paper considers an example of automation of radio measurement tools within the educational process. An example of the development of a digital curve tracer, used in laboratory experiments when removing the voltageampere characteristics of nonlinear radio elements (semiconductor devices) is given. The method of statistical processing of measurement results in Microsoft Office Excel is proposed.

Key words: Digitalization of measuring instruments, curve tracer, volt-ampere characteristic, semiconductor diodes, digital modeling.

При подготовке специалистов по технической эксплуатации транспортного радиооборудования большое значение уделяется лабораторным занятиям с постановкой натурных экспериментов, что позволяет студентам освоить профессиональные компетенции: «Способен проводить измерения и инструментальный контроль, проводить обработку результатов и оценивать погрешности», «Способен применять естественнонаучные и общеинженерные знания, аналитические методы в профессиональной деятельности».

На начальном этапе формирования индикаторов компетенций у студентов необходимо отработать навыки снятия технических параметров радиотехнических элементов и систем, одним из которых является вольтамперная характеристика (BAX) нелинейных радиоэлементов (например, диодов).

В дисциплинах «Физические основы электроники», «Теоретические основы электротехники» поставлена методика выполнения лабораторных работ, позволяющая провести натурный эксперимент по получению $\mathrm{BAX}$ диодов с использованием мультиметров (рис. 1).

Студенты измеряют напряжения и токи при прямом и обратном включении диода. Потом строят графики. Данная методика снятия ВАХ является очень трудоемкой, так как для качественного построения графика кривой $\mathrm{BAX}$ в нелинейных цепях требуется большое количество точек. Поэтому на кафедре радиоэлектроники ФГБОУ ВО «ВГУВТ» в рамках занятий студенческого научного общества предложена автоматизация процесса радиоизмерений при снятии ВАХ полупроводниковых элементов $[1,2]$. 

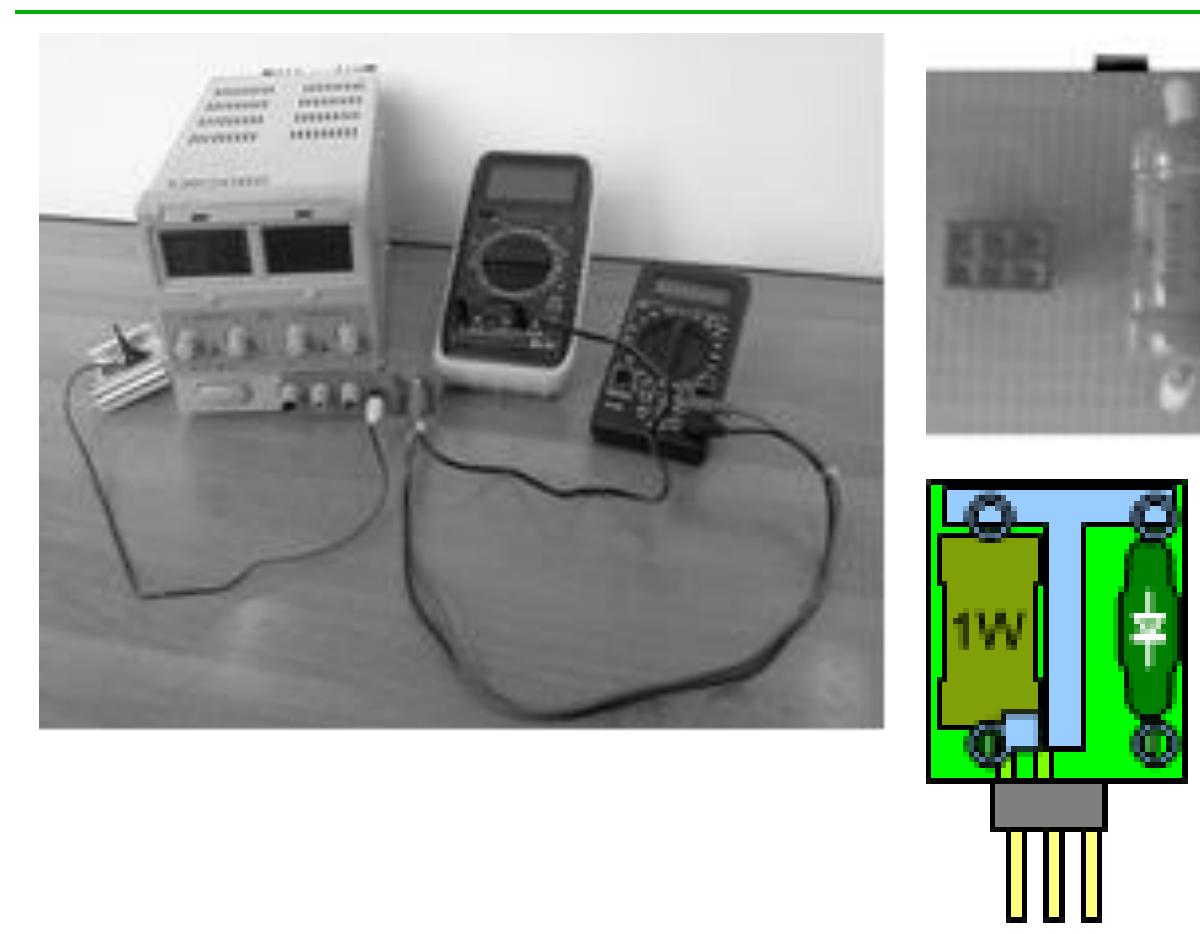

Рис. 1. Лабораторная установка для исследования вольт-амперных характеристик p-n переходов: общий вид установки; увеличенная фотография объединительной платы, сменный модуль для диода малой мощности

Для снижения трудоемкости и повышения точности результатов в радиоизмерениях применяют автоматизированные измерительные приборы характериографы, выполненные на цифровой элементной базе [3].

Для автоматизации процесса снятия ВАХ полупроводниковых приборов на кафедре радиоэлектроники был разработан и собран характериограф на базе аппаратно-программной платформы Arduino Nano v.3 и модулей на микросхемах ADC1115 (аналого-цифровой преобразователь, используемый в качестве считывающего устройства блока измерений) и MCP4725 (цифроаналоговый преобразователь, используемый в качестве источника опорного напряжения (ИОН)). Структура лабораторной установки приведена на рис.2 [4]. 


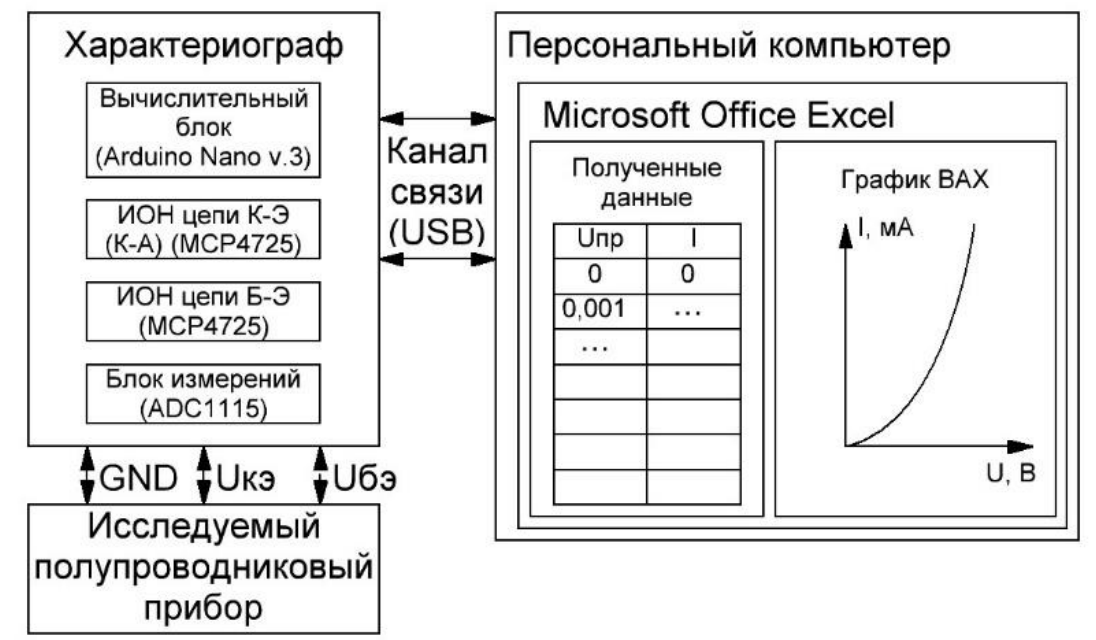

Рис. 2. Структурная схема лабораторной установки по измерению ВАХ полупроводниковых приборов

В результате измерений ВАХ полупроводникового прибора (диод Д220) получена таблица значений приложенных к прибору напряжений и протекающих через него токов [4].

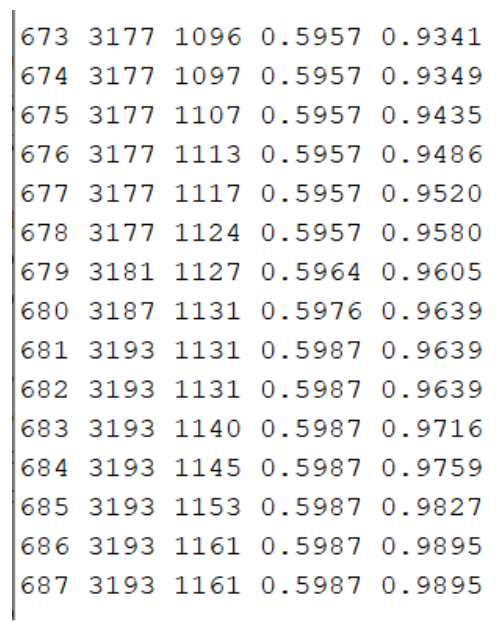

\section{Рис. 3. Пример фрагмента таблицы данных с характериографа}

Для построения ВАХ НЭ по данным с характериографа может быть получено до 4095 точек. Цикл измерения (получения точки) происходит следующим образом: согласно номеру текущей итерации измерения (показан на рис.3 первый столбец) модуль ЦАП формирует испытательное напряжение, которое подается на цепь, состоящую из измерительного сопротивления и 
исследуемого полупроводникового прибора. При подаче напряжения через цепь протекает ток, который, в свою очередь, вызывает падение напряжения на измерительном сопротивлении. Значения напряжения на выходе ИОН (до сопротивления) и на выводе исследуемого прибора (после сопротивления) измеряются, результаты измерений обрабатываются микроконтроллером: фильтруются от помех, вычисляются битовые значения приложенного напряжения (рис.3 столбец 2), битовое значение протекающего тока (рис.3 столбец 3). Используя заданные коэффициенты пропорциональности, вычисляются реальные значения приложенного напряжения в вольтах (рис.3 столбец 4), и реальное значение протекающего тока в миллиамперах (рис.3 столбец 5). При фильтрации собирается выборка из восьми измерений. Количество измерений в выборке фильтрации, а также количество итоговых точек, от которых зависит точность и время измерения BAX могут быть программно настроены.

Исследование свойств нелинейных элементов и цепей в радиотехнике проводится с помощью математических моделей, причем при расчетах предпочтительно аппроксимировать измеренные данные BAX не графической, а полиномиальной характеристикой $[5,6]$.

Для дальнейшей обработки полученных данных используется пакет Microsoft Office Excel. По полученным с характериографа данным строится кривая BAX диода. Пакет Microsoft Office Excel позволяет для выбранного графика построить линию тренда и провести ее полиномиальную аппроксимацию. График ВАХ описывается полиномом Лагранжа, величина достоверности аппроксимации зависит от выбранной степени аппроксимирующего полинома. ВАХ прибора описывается уравнением:

$$
i_{\mathrm{Bdx}}=a_{0}+a_{1} U_{\mathrm{BX}}+a_{2}{U_{\mathrm{Bx}}}^{2}+a_{3}{U_{\mathrm{Bx}}}^{3}+\cdots+a_{n} U_{\mathrm{BX}}{ }^{n},
$$

где: $i_{\text {вых }}$ - выходной ток прибора (диода, транзистора), $U_{\text {вх }}-$ входной сигнал (мгновенное значение приложенного напряжения), коэффициенты $a_{1}-$ $a_{n}$ играют роль проводимостей.

Для учета влияния BAX реальных приборов на параметры преобразуемых цепями сигналов коэффициенты полинома определяются по уравнению кривой, полученной при натурном эксперименте характериографом.

При аппроксимации кривой ВАX полиномом второго порядка (выражение (2)) линия тренда (сплошная линия черного цвета на рис. 4) не 
совпадает с кривой, построенной по экспериментальным данным (пунктир на рис. 4), то есть, достоверность аппроксимации недостаточна.

$$
i_{\text {вых }}=4,1982-36,999 \cdot U_{\mathrm{BX}}+51,907 \cdot U_{\mathrm{BX}}{ }^{2} \text {. }
$$

При повышении степени аппроксимирующего полинома (пятая степень выражение (3)), возрастает величина достоверности аппроксимации, см. рис.5 (графики экспериментальной ВАХ и линии тренда практически совпадают).

$$
i_{\mathrm{Bdx}}=-0,1125+4,0258 \cdot U_{\mathrm{BX}}-30,22 \cdot U_{\mathrm{BX}}{ }^{2}+86,34 \cdot U_{\mathrm{BX}}{ }^{3}-
$$

$123,25 U_{\mathrm{BX}}{ }^{4}++89,315 \cdot U_{\mathrm{BX}}{ }^{5}$

Уравнение кривой линии тренда отображает математическую модель BAX прибора: где по оси у - ток через прибор, по оси $\mathrm{x}$ - приложенное к прибору мгновенное значение напряжения.

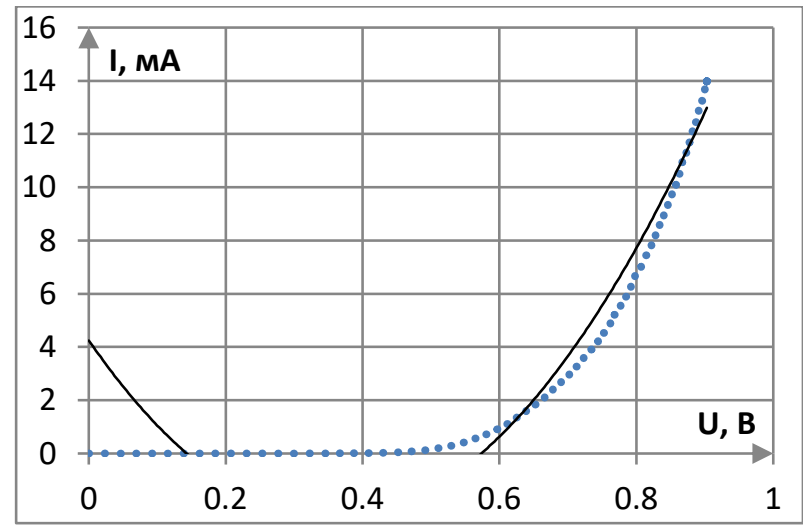

Рис. 4. ВАХ диода Д220 при аппроксимации полиномом второго порядка

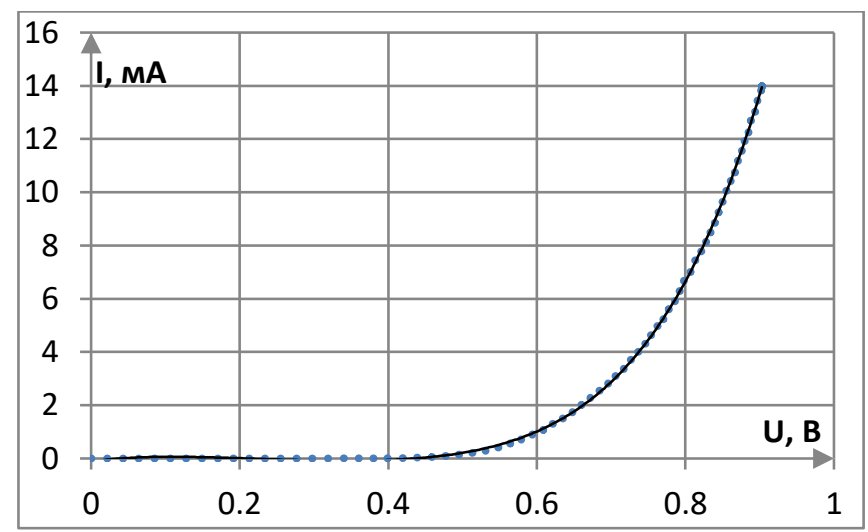

Рис. 5. ВАХ диода Д220 при аппроксимации полиномом пятого порядка

Рассмотренные примеры синтеза модели ВАХ нелинейных элементов с разными степенями аппроксимирующих полиномов позволяют определиться исследователю с уровнем детализации математической модели изучаемого объекта в зависимости от условий решаемой задачи.

Внедрение разработанного цифрового характериографа и методики статистической обработки результатов измерений $\mathrm{BAX}$ нелинейных радиотехнических цепей в учебный процесс позволит синтезировать более детальные математические модели и эффективнее исследовать технические параметры объектов профессиональной деятельности. 


\section{Список литературы}

1. Мельников М.А., Гордяскина Т.В. Исследование нелинейных аналоговых радиотехнических цепей (амплитудных модуляторов) в программном пакете mathcad. //Великие реки 2020: Материалы международной научно-методической конференции. ФГБОУ ВО «ВГУВТ». - 2020. - URL: http://вф-река-море.pф/2020/PDF/9_9.pdf (дата обращения 19.10.2021).

2. Баскаков С.И. Радиотехнические цепи и сигналы : учебник. - М. :Высш. Школа, 2000. - 462 с.

3. Fatih Önder, Esra Bìlal Önder, and Mehmet Oğur "Determination of Diode Characteristics by Using Arduino" Phys. Teach. 57, 244-245 (2019). https://doi. org/10.1119/1.5095382 (дата обращения 19.10.2021)

4. Мельников М. А. Разработка автоматизированных средств измерения (характериографов) на базе аппаратно-программной платформы Arduino//XXVI Нижегородская сессия молодых ученых (технические и естественные науки): Сборник сессии молодых ученых (технические и естественные науки). ГБУ ДПО "Нижегородский научно-информационный центр" - 2021. - URL: https://www.elibrary.ru/item.asp?id=46551653\&pff=1 (дата обращ.22.09.2021).

5. Мартынов Н.С., Гордяскина Т.В. Исследование нелинейных аналоговых радиотехнических цепей (амплитудных модуляторов) в программном пакете Multisim. Труды конгресса «Великие реки» 2019 Выпуск 8, 2019г. URL:http://вф-река-море.pф/2019/PDF/9_5.pdf (дата обращения 19.04.2021).

6. Панков Е.А., Мартынов Н.С., Гордяскина Т.В., Лебедева С.В. Техническая диагностика нелинейных аналоговых радиотехнических систем в программном пакете Multisim. Труды конгресса «Великие реки» 2019 Выпуск 8, 2019г. URL:http://вф-река-море.pф/2019/PDF/9_7.pdf (дата обращ. 19.04.2021). 